\title{
I solated hyperphosphatemia as an early manifestation in asymptomatic multiple myeloma
}

\author{
Pritam Singh, Monica Gupta \\ Government Medical College and Hospital, Chandigarh, India
}

Correspondence: Monica Gupta. Address: Government Medical College and Hospital, Sector 32, Chandigarh-160030, India. Email: monicamanish2001@gmail.com

Received: January 20, 2014

Accepted: March 4, $2014 \quad$ Online Published: March 10, 2014

DOI : $10.5430 /$ crim.v1n2p1

URL: http://dx.doi.org/10.5430/crim.v1n2p1

\begin{abstract}
Usual presentation of multiple myeloma with symptoms like bone pains, renal failure or recurrent infections do not pose much of a diagnostic challenge, but difficulties arise when symptoms are vague and spurious results of laboratory investigations complicate the clinical picture. Hyperphosphatemia is usually observed in cases that have high disease burden (tumor lysis syndrome) or impaired renal functions; however its occurrence with solitary plasmacytoma or early stages (low burden) with preserved renal functions is unlikely and probably best explicable on account of pseudohyperphosphatemia- a laboratory error that results from interference in colorimetric estimation of serum phosphate because of existence of paraproteins in these patients. We, hereby, report a case where evaluation of asymptomatic hyperphosphatemia led to the final diagnosis of multiple myeloma.
\end{abstract}

\section{Keywords}

Pseudohyperphosphatemia, Multiple myeloma

\section{I ntroduction}

Multiple myeloma represents malignant proliferation of plasma cells derived from a single clone. The disease usually presents with bone pains or fracture, renal failure, susceptibility to infections, anemia and hypercalcemia. Occurrence of hyperphosphatemia in multiple myeloma is not well established in patients with preserved renal functions ${ }^{[1]}$. It may be detected in patients with high disease burden, where multiple lytic lesions may contribute to increased phosphate delivery to plasma, but hyperphosphatemia in solitary lesions (solitary plasmacytoma) is uncommon; however such occurrences of hyperphosphatemia can be reasoned on account of pseudohyperphosphatemia, which is due to paraproteinemia that interferes with the colorimetric estimation of serum phosphate levels ${ }^{[1,2]}$.

\section{Case presentation}

A 45-year-old male presented to Medicine outpatient department with the lone complaint of decreased appetite for the past one and a half month. There was no history of associated abdominal pains, vomiting, altered bowel habits, gastro-esophageal reflux, jaundice or fever. There was no history of any significant weight loss, pain in bones or joints. There were no other systemic complaints like chronic cough, expectoration, chest pain, dyspnea, orthopnea, palpitations or 
easy fatigability. He had no significant past medical history and was otherwise healthy. Patient was not a known diabetic or hypertensive. Patient was a non-smoker but consumed alcohol (about $30 \mathrm{ml} /$ day of $40 \%$ alcohol for last 2 years). There was no history of illicit drug abuse, high-risk sexual behavior or any psychogenic stressor. Patient belonged to lower socio-economic strata. On examination patient was of average built and nutrition. His vitals were stable and systemic examination was notable for absence any significant positive findings.

As a preliminary approach a routine metabolic profile was performed and it showed normal blood counts with hemoglobin $12.8 \mathrm{~g} / \mathrm{dl}$ and TLC 8,700/cc with DLC as polymorphs 68\% lymphocytes 30\% and monocytes $2 \%$ but elevated ESR (75 $\mathrm{mm}$ in 1st hour, Westergren method). Liver function test showed serum bilirubin level $0.8 \mathrm{mg} / \mathrm{dL}$ and normal AST (25 $\mathrm{U} / \mathrm{L})$ and ALT (30 U/L) but hypoalbuminemia $(2 \mathrm{~g} / \mathrm{dL})$ with normal total proteins $(7 \mathrm{~g} / \mathrm{dl})$. Renal function tests revealed blood urea of $26 \mathrm{mg} / \mathrm{dL}$ and serum creatinine of $0.8 \mathrm{mg} / \mathrm{dL}$, however there was marked hyperphosphatemia $(>10 \mathrm{mg} / \mathrm{dL})$ with normal serum calcium levels $(10.3 \mathrm{mg} / \mathrm{dL})$. Patient's chest X-ray was normal and USG abdomen revealed bilaterally normal sized kidneys with maintained corticomedullary differentiation. There was no evidence of hydronephrosis. Patient was advised admission for further work up of marked hyperphosphatemia with normal serum calcium levels.

However patient lost to follow up only to return back 3 months later with spastic paraparesis. Again routine investigations showed a similar profile with persistent hyperphosphatemia. MRI of spine was performed which showed a collapse of D11 vertebra with hyperintense marrow signal on T-2 weighted images with consequent posterior bulge of the vertebral body leading to compression of thecal sac and compression of the spinal cord. Intervertebral disc spaces were normal in morphology and signal pattern. It was at this point, with a clinical picture of vertebral collapse, raised ESR and hyperphosphatemia that the possibility of multiple myeloma was considered. Patient's skeletal survey was done which did not show any evidence of lytic lesions elsewhere. Thereafter patient's bone marrow aspiration was done to rule out disseminated tuberculosis or multiple myeloma and it revealed increased bone marrow plasma cells (16\%), serum protein electrophoresis showed A/G reversal (0.28) and hypergammaglobulinemia (64\%) with M-spike. On immunofixation IgG and $\lambda$ chains were found. Serum IgG levels were estimated to be $3.8 \mathrm{~g} / \mathrm{dL}$ and thus classified as stage I of multiple myeloma as per the Durie and Salmon staging system. Finally patient was referred to tertiary care centre with facility for oncology consultation and palliative radiotherapy.

\section{Discussion}

Hyperphosphatemia is a known complication related to renal dysfunction and high disease burden in cases of multiple myeloma, however initial presentation with unexplained hyperphosphatemia leading to a diagnosis of multiple myeloma is a rare occurrence; more so in cases with low disease burden (early stages) ${ }^{[1,2]}$. The occurrence of hyperphosphatemia in these patients has been attributed to large-scale use of ammonium molybdate as a reagent in the commercial diagnostic kits routinely available for estimation of serum phosphate level ${ }^{[3,4]}$. The interaction between the reagent and myeloma proteins increases the optical density of the test sample and thus falsely high measured serum inorganic phosphate levels are recorded (pseudohyperphosphatemia). Since we used a similar diagnostic kit with ammonium molybdate as a reagent, the possibility of pseudohyperphosphatemia cannot be ruled out. Other endocrinal causes of hyperphosphatemia are always associated with counter changes in serum calcium levels. Authors concluded that the index case had pseudohyperphosphatemia as there were no counter-changes in serum calcium levels and renal functions were normal. Hence, in all likelihood patient had pseudohyperphosphatemia. By reporting this case, we want to draw attention of our colleagues that isolated hyperphosphatemia can be an earliest and asymptomatic presentation of early stages of multiple myeloma.

Thus, such a picture of marked and isolated hyperphosphatemia without counter-changes in serum calcium levels and normal kidney function tests on laboratory investigations should draw the attention of the treating physician to the possibility of pseudo-hyperphosphatemia to avoid unnecessary investigations and lead to a directed evaluation of the patient to its potential causes. Moreover, while dealing with such cases the physician should rule out pseudohyperphosphatemia, which can be done by using diagnostic kits where serum proteins are removed from the test sample by 
dialysis prior to addition of the reagent such as done in Vanadate method ${ }^{[3]}$. The urgency to reach a final diagnosis in such a condition is justified by the fact that the occurrence of hyperphosphatemia in multiple myeloma has been known to be associated positively with other poor prognostic markers such as presence of b-2 microglobulins, increased serum calcium, increased serum uric acid, increased serum creatinine, decreased hemoglobin and increased percentage of plasma cells in bone marrow etc ${ }^{[5]}$. Authors emphasize on pseudohyperphosphatemia as the first sign because if pseudohyperphosphatemia was recognized and evaluated early, this could have prevented a catastrophic event like vertebral collapse and compressive myelopathy in our patient. Sensitization to this aspect may help physicians in early diagnosis and benefit patients from avoiding such complications. Also the knowledge of this phenomenon of pseudo-hyperphosphatemia may avoid confusion, unnecessary investigations in clinical evaluations of the patients. Another point of interest is that a laboratory investigation such as hyperphosphatemia, irrespective of whether true or pseudohyperphosphatemia may precede other clinical manifestations of multiple myeloma with sufficient temporal profile so as to serve as a surrogate marker of the disease.

\section{References}

[1] Mandry JM, Posner MR, Tucci JR, Eid C. Hyperphosphatemia in multiple myeloma due to phosphate-binding immunoglobulin.Cancer. 1991; 68(5): 1092-1094. http://dx.doi.org/10.1002/1097-0142(19910901)68:5<1092::AID-CNCR2820680531>3.0.CO;2-I

[2] Busse JC, Gelbard MA, Byrnes JJ, Hellman R, Vaamonde CA. Pseudohyperphosphatemia and dysproteinemia. Arch Intern Med. 1987; 147(11):2045-2046. http://dx.doi.org/10.1001/archinte.1987.00370110173027

[3] Sonnenblick M, Eylath U, Brisk R, Eldad C, Hershko C. Paraproteininterference with colorimetry of phosphate in serum of some patients of myeloma. Clin Chem. 1986; 32: 1537-1539.

[4] Barutcuoglu B, Parildar Z, Mutaf I, Habif S, Bayindir O. Spuriously elevated inorganic phosphate level in a patient of multiple myeloma. Clin Lab Haematol. 2003; 25: 271-274. http://dx.doi.org/10.1046/j.1365-2257.2003.00524.x

[5] Umeda M, Okuda S, Izumi H, Nagase D, Fujimoto Y, Sugasawa Y, et al. Prognostic significance of the serum phosphorus level and its relationship with other prognostic factors. Ann Hematol. 2006; 85: 469-473. http://dx.doi.org/10.1007/s00277-006-0095-3 